\title{
Third generation EGFR inhibitor osimertinib combined with pemetrexed or cisplatin exerts long-lasting anti-tumor effect in EGFR-mutated pre-clinical models of NSCLC
}

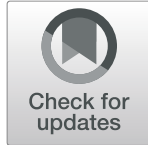

Silvia La Monica ${ }^{1}$, Roberta Minari ${ }^{2}$, Daniele Cretella ${ }^{1}$, Lisa Flammini ${ }^{3}$, Claudia Fumarola ${ }^{1}$, Mara Bonelli ${ }^{1}$, Andrea Cavazzoni', Graziana Digiacomo ${ }^{1}$, Maricla Galetti ${ }^{4,5}$, Denise Madeddu', Angela Falco', Costanza Annamaria Lagrasta ${ }^{1}$, Anna Squadrilli ${ }^{2}$, Elisabetta Barocelli ${ }^{3}$, Alessandro Romanel ${ }^{6}$, Federico Quaini ${ }^{1}$, Pier Giorgio Petronini ${ }^{1}$, Marcello Tiseo ${ }^{1,2^{*}+}$ and Roberta Alfieri ${ }^{{ }^{* *}}$ (D)

\begin{abstract}
Background: The third generation Epidermal Growth Factor Receptor (EGFR) Tyrosine Kinase Inhibitor (TKI) osimertinib has been initially approved for T790M positive Non-Small Cell Lung Cancer (NSCLC) and more recently for first-line treatment of EGFR-mutant T790M negative NSCLC patients. Similarly to previous generation TKIs, despite the high response rate, disease progression eventually occurs and current clinical research is focused on novel strategies to delay the emergence of osimertinib resistance. In this study we investigated the combination of osimertinib with pemetrexed or cisplatin in EGFR-mutated NSCLC cell lines and xenografts.
\end{abstract}

Methods: Tumor growth was evaluated in a PC9T790M xenograft model and tissue composition was morphometrically determined. PC9, PC9T790M and HCC827 cell lines were employed to test the efficacy of osimertinib and chemotherapy combination in vitro. Cell viability and cell death were evaluated by MTT assay and fluorescence microscopy. Protein expression and gene status were analysed by Western blotting, fluorescence in situ hybridization analysis, next-generation sequencing and digital droplet PCR.

Results: In xenograft models, osimertinib significantly inhibited tumor growth, however, as expected, in $50 \%$ of mice drug-resistance developed. A combination of osimertinib with pemetrexed or cisplatin prevented or at least delayed the onset of resistance. Interestingly, such combinations increased the fraction of fibrotic tissue and exerted a long-lasting activity after stopping therapy. In vitro studies demonstrated the stronger efficacy of the combination over the single treatments in inhibiting cell proliferation and inducing cell death in PC9T790M cells as well as in T790M negative PC9 and HCC827 cell lines, suggesting the potential role of this strategy also as first-line treatment. Finally, we demonstrated that osimertinib resistant clones, either derived from resistant tumors or generated in vitro, were less sensitive to pemetrexed prompting to use a chemotherapy regimen non-containing pemetrexed in patients after progression to osimertinib treatment.

Conclusions: Our results identify a combination between osimertinib and pemetrexed or cisplatin potentially useful in the treatment of EGFR-mutated NSCLC patients, which might delay the appearance of osimertinib resistance with longlasting effects.

Keywords: Non-Small Cell Lung Cancer, Osimertinib, Resistance, Epidermal Growth Factor Receptor, Pemetrexed, Cisplatin

\footnotetext{
*Correspondence: marcello.tiseo@unipr.it; roberta.alfieri@unipr.it

${ }^{+}$Marcello Tiseo and Roberta Alfieri contributed equally to this work.

'Department of Medicine and Surgery, University of Parma, Parma, Italy

Full list of author information is available at the end of the article
}

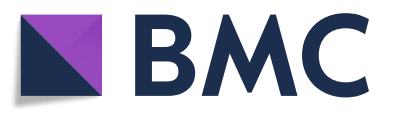

(c) The Author(s). 2019 Open Access This article is distributed under the terms of the Creative Commons Attribution 4.0 International License (http://creativecommons.org/licenses/by/4.0/), which permits unrestricted use, distribution, and reproduction in any medium, provided you give appropriate credit to the original author(s) and the source, provide a link to the Creative Commons license, and indicate if changes were made. The Creative Commons Public Domain Dedication waiver (http://creativecommons.org/publicdomain/zero/1.0/) applies to the data made available in this article, unless otherwise stated. 


\section{Background}

Epidermal Growth Factor Receptor (EGFR) mutationpositive lung cancer occurs in about $10-15 \%$ of Caucasian patients and $40-50 \%$ of Japanese patients. NonSmall Cell Lung Cancer (NSCLC) patients harboring EGFR activating mutations, such as in-frame deletions in exon 19 (Ex19del) or missense mutation in exon 21(L858R), show high sensitivity to EGFR tyrosine kinase inhibitors (TKIs) such as gefitinib, erlotinib, afatinib and dacomitinib. However, the acquisition of EGFR T790M secondary mutation, the substitution of threonine 790 with methionine, is responsible for half of the cases of acquired resistance to TKI treatment [1].

Osimertinib, a third-generation EGFR-TKI that selectively binds the $\mathrm{C} 797$ residue inhibiting the T790M mutation, has shown high activity in term of Progression-Free Survival (PFS) and overall response rate in EGFRT790M positive patients [2,3] and efficacy superior to gefitinib/erlotinib in the first-line treatment by approximately a 9 months advantage in PFS [4]. However, acquired resistance occurs also to osimertinib either in T790M-positive NSCLC patients or in patients treated in first-line $[5,6]$. EGFR-dependent or -independent mechanisms of resistance have been described even if they remain not completely understood [5]. EGFR G796/ C797, L792 and L718/G719 mutations, MET and HER2 amplification, BRAF, KRAS, and PIK3CA mutations, oncogenic fusion mutations in FGFR3, RET, and NTRK were recently identified in a large cohorts of osimertinib-resistant lung cancer patients either treated in second-line [7, 8] and in first-line [9]. Knowledge of these mechanisms is relevant in order to develop new therapeutic strategies to overcome TKI-resistance; however, how prevent or delay the acquisition of resistance remains an important issue. Our previous data indicated that in PC9 cell line and xenograft models the combination of gefitinib with pemetrexed or the intermittent combination of pemetrexed and gefitinib prevented the appearance of gefitinib resistance mediated by T790M mutation and epithelial-mesenchymal transition [10]; however, the combination was ineffective when gefitinib was administered before pemetrexed. Theoretically, chemotherapy, given its different and more generic mechanism of action, can postpone the resistance to EGFRTKIs by limiting the tumor heterogeneity, thus improving the efficacy of treatment either in first- and second-line.

In view of this recent very favorable experience, osimertinib combined or intercalated with chemotherapy deserves to be considered either for patients in progression after first/second-generation TKIs or in first-line setting. Neither preclinical nor clinical data are available to date.

Pemetrexed-platinum based chemotherapy remains the standard of care for T790M negative patients progressing after first-line EGFR-TKIs and for patients with
T790M positive tumors in progression after second-line osimertinib [11].

Our study was undertaken to explore the combination of osimertinib with pemetrexed or cisplatin in vivo in a mouse model of PC9T790M xenograft and in vitro in PC9T790M, PC9 and HCC827 cell lines.

\section{Methods \\ Cell lines and culture}

The NSCLC cell line PC9, harboring an in-frame deletion in exon 19 of EGFR gene, was kindly provided by Dr. P. Jänne (Dana-Farber Cancer Institute, Boston MA). PC9T790M cell clone was generated by exposing PC9 parental cells to increasing concentrations of gefitinib [10]. This cell clone was cultured in the presence of gefitinib $1 \mu \mathrm{M}$ to maintain a selection pressure during in vitro propagation. HCC827 cell line was from ATCC (Manassas, VA). PC9T790M clones resistant to osimertinib were isolated after 9 months of culturing PC9T790M cells in the presence of increasing concentrations of osimertinib (from $10 \mathrm{nM}$ to $500 \mathrm{nM}$ ). Clones were cultured in the presence of $500 \mathrm{nM}$ osimertinib. Cells were cultured in RPMI-1640 (Life Technologies, Gaithersburg, MD) medium supplemented with $10 \%$ fetal bovine serum (Life Technologies) and maintained under standard cell culture conditions at $37^{\circ} \mathrm{C}$ in a water-saturated atmosphere of $5 \% \mathrm{CO}_{2}$ in air.

\section{Drug treatment}

Osimertinib was provided by AstraZeneca (Milan, Italy). Pemetrexed and cisplatin were from inpatient pharmacy of University Hospital of Parma. Osimertinib was dissolved in DMSO (Sigma, ST Louis, MO), while pemetrexed and cisplatin were dissolved in $0.9 \%$ sodium chloride solution and diluted in fresh medium before use. Final DMSO concentration in medium never exceeded $0.1 \%(\mathrm{v} / \mathrm{v})$ and equal amounts of the solvent were added to control cells.

\section{Analysis of cell proliferation, cell viability and cell death} Cell number, cell viability and cell death were evaluated as previously described [12].

\section{Western blot analysis}

Procedures for protein extraction, solubilization, and protein analysis by 1-D PAGE are described elsewhere [13]. Antibody against Thymidylate Synthase (TS) was from Upstate (Lake Placid, NY); antibody against MET, p-EGFRTyr1068, EGFR, pAKTser473, AKT, p-ERK1/ 2Thr202/Tyr204, ERK1/2, Bim, cleaved caspase-7, PARP and HRP-conjugated secondary antibodies was from Cell Signaling Technology (Beverly, MA); antibody against Actin was from Sigma; and chemoluminescence system (ImmobilionTM Western Chemiluminescent HRP 
Substrate) was from Millipore (Temecula, CA). Reagents for electrophoresis and blotting analysis were from BIORAD (Hercules, CA).

\section{Tumor xenografts}

A total of $5 \times 10^{6}$ PC9T790M cells were suspended in $200 \mu \mathrm{L}$ of Matrigel (BD Biosciences, Erembodegem, Belgium)/PBS (1:1) and were subcutaneously injected in the flank of Balb/c-Nude female mice (Charles River Laboratories, Calco, Italy). The animals were housed in a protected unit for immunodeficient animals with 12-h light-dark cycles and provided with sterilized food and water ad libitum. When tumor volume reached an average size of $150 \mathrm{~mm}^{3}$, animals $(N=8)$ were treated with osimertinib ( $3 \mathrm{mg} / \mathrm{kg}$ in $1 \%$ Tween 80 ) given once per day, five times per week, by oral gavage; pemetrexed $(100 \mathrm{mg} / \mathrm{kg}$ in $0.9 \% \mathrm{NaCl})$ [10], cisplatin $(4 \mathrm{mg} / \mathrm{kg}$ in $0.9 \% \mathrm{NaCl}$ ) [14] or vehicle alone (control group) were administered intraperitoneally once a day, twice per week. Tumor xenografts were measured as previously described [15]. At the end of the experiments, mice were euthanized by cervical dislocation and tumors weighted and collected for immunohistochemical and nextgeneration sequencing (NGS) analysis.

All experiments involving animals and their care were performed with the approval of the Local Ethical Committee of University of Parma (Organismo per la Protezione e il Benessere degli Animali) and by the Italian Ministry of Health, in accordance with the institutional guidelines that are in compliance with national (D.Lgs. 26/2014) and international (Directive 2010/63/EU) laws and policies.

\section{Isolation and in vitro expansion of neoplastic cells from xenograft tumors}

Tissues from osimertinib-resistant tumors were enzymatically digested using the Tumor Dissociation Kit from Miltenyi Biotec (Bergisch Gladbach, Germany) and the gentleMACS ${ }^{\mathrm{mm}}$ Dissociator (Miltenyi Biotec) was used for the mechanical dissociation. The single-cell suspension obtained after digestion was enriched in tumor cells using the Tumor Cell Isolation Kit (Miltenyi Biotec) accordingly to manufacturer's instructions, as previously described [10]. Tumor cells were cultured with $500 \mathrm{nM}$ osimertinib to maintain a selection pressure during in vitro propagation.

\section{Morphometric analysis of tumor xenografts}

Subcutaneous nodules were excised, formalin fixed, paraffin embedded and processed for histochemical analysis. The morphometric evaluation of xenograft composition was performed on Masson's trichrome-stained sections. In detail, the number of points overlying neoplastic tissue, fibrosis or necrosis was counted and expressed as percentage of the total number of points explored to define the volume fractions of each tissue component. All these morphometric measurements were obtained with the aid of a grid defining a tissue area of $0.22 \mathrm{~mm}^{2}$ and containing 42 sampling points each covering an area of $0.0052 \mathrm{~mm}^{2}$. These evaluations were performed on the entire section of each tumor sample using an optical microscope (200X final magnification).

\section{Fluorescence in situ hybridization (FISH) analysis}

FISH analysis of EGFR, MET, and HER2 genes was assessed on histologic samples to evaluate their amplification within the tumor xenografts. Briefly, following DNA denaturation, specific fluorescent probes, complementary to the analyzed genes and to the centromeric region of the chromosome (CEP), were applied and sections were post-hybridized according to manufacturer's instructions. Nuclei were counterstained with DAPI, and the images were acquired and analyzed at $1000 \mathrm{X}$ magnification using a fluorescence microscope (Nikon) with Z-stack equipment. Tumors were defined amplified if EGFR/CEP7, MET/CEP7, HER2/CEP17 ratio was $\geqslant 2$.

\section{Next-generation sequencing and digital droplet PCR}

NGS was performed with Solid Tumor SolutionTM (Sophia Genetics) on the MiSeq platform (Illumina ${ }^{\circ}$, San Diego, CA). Genomic DNA was extracted from tumor nodules with Qiagen DNA Mini Kit (Qiagen ${ }^{\circ}$, Valencia, CA, USA) and quantified with QuantiFluor ${ }^{\circ}$ dsDNA System (Promega). Each libraries were prepared starting from $65 \mathrm{ng}$ of genomic DNA, in according to manufacturer's protocol. 10pM of diluted and denatured libraries were loaded on MiSeq Reagent Kit v3. Data were analyzed with Sophia DDM platform (Sophia Genetics). This NGS panel allowed to provide a comprehensive assessment of Single Nucleotide Variation (SNV) and Indels in hotspot of 42 genes ( $A K T 1, A L K, B R A F, C D K 4$, CDKN2A, CTNNB1, DDR2, DICER1, EGFR, ERBB2, ERBB4, FBXW7, FGFR1, FGFR2, FGFR3, FOXL2, GNA11, GNAQ, GNAS, H3F3A, H3F3B, HIST1H3B, HRAS, IDH1 IDH2, KIT, KRAS, MAP $2 \mathrm{~K} 1$, MET, MYOD1, NRAS, PDGFRA, PIK3CA, PTPN11, RAC1, RAF1, RET, ROS1, SF3B1, SMAD4, TERT,TP53) and gene amplification events over 24 genes which are $A L K$, BRAF, CDK4, CDKN2A, EGFR, ERBB2, FBXW7, FGFR1, FGFR2, FGFR3, HRAS, KIT, KRAS, MET, MYOD1, NRAS, PDGFRA, PIK3CA, RAF1, ROS1, RET, SF3B1, TERT and TP53.

Digital droplet PCR (ddPCR) was performed on tumor DNA to assess the presence of tertiary EGFR mutation. Site specific mutation detection assays were performed to study EGFR C797, L792 and L718 mutations and copy number assays were performed to assess the amplification 
of EGFR and NRAS as a potential mechanism of resistance.

\section{Statistical analysis}

Statistical analyses were carried out using GraphPad Prism version 6.0 software (GraphPad Software Inc., San Diego, CA). Results are expressed as mean values \pm standard deviations (SD) for the indicated number of independent measurements. Differences between the mean values recorded for different experimental conditions were evaluated by Student's t-test or by one-way ANOVA followed by Bonferroni's post-test, and $p$ values are indicated where appropriate in the figures and in their legends. $P$ values $<0.05$ were considered as significant. For in vivo studies comparison among groups was made using two-way repeated measures ANOVA followed by Bonferroni's post-test (to adjust for multiple comparisons). Adjusted $p$ values of less than 0.05 were considered significant.

\section{Results}

Efficacy of osimertinib combined with pemetrexed or cisplatin on tumor growth in PC9T790M xenograft and cell line models

Firstly, the effects of the combination of osimertinib with pemetrexed were investigated on PC9T790M (EGFR exon 19 E746-A750 deletion, T790M positive) xenograft models.

PC9T790M cells were subcutaneously inoculated into Balb/c-Nude female mice and after tumors had reached an average size of about $150 \mathrm{~mm}^{3}$ the animals were randomized into four different groups: vehicle alone (ctrl); osimertinib (osi), osimertinib intercalated, every week, with pemetrexed (pem) (osi $\rightarrow$ pem); and osimertinib plus pemetrexed intercalated, every week, with osimertinib alone (osi + pem $\rightarrow$ osi). As illustrated in Fig. 1a, the continuous osimertinib treatment inhibited tumor growth up to 50 days. Subsequently, four of eight animals treated with osimertinib alone developed acquired resistance. When osimertinib was intercalated with pemetrexed (osi $\rightarrow$ pem) the inhibition of tumor growth was observed for 30 days, after which all the tumors started to grow again. Conversely, in the group of animals treated with osimertinib plus pemetrexed intercalated, every week, with osimertinib alone (osi + pem $\rightarrow$ osi), tumor regression was observed all along the experiment in all mice. Tumor growth was monitored for 110 days and during this period mice showed no signs of toxicity and regularly gained body weight (Fig. 1b).

In order to test the treatment efficacy in tumor eradication, osimertinib or osimertinib combined with pemetrexed treatments were stopped and regrowth of small tumors was monitored. All four small tumors from osimertinib monotherapy rapidly relapsed, while no relapses were observed when osimertinib had been coadministered with pemetrexed (Fig. 1c, d).

Subsequently, we tested whether also cisplatin may exert a persistent tumor-inhibitory effect in PC9T790M xenografts when combined with osimertinib. PC9T790M cells were subcutaneously inoculated into Balb/c-Nude female mice and after tumors had reached an average size of about $150 \mathrm{~mm}^{3}$ the animals were randomized into three different groups: vehicle alone (ctrl); osimertinib (osi); and osimertinib plus cisplatin (cis) intercalated, every week, with osimertinib alone (osi + cis $\rightarrow$ osi). As shown in Fig. 2a, osimertinib treatment inhibited tumor growth up to 45 days, then acquired resistance developed in four of the eight animals. In contrast, a significant inhibition of tumor growth was observed in all mice treated with osimertinib combined with cisplatin intercalated, every week, with osimertinib alone. However, differently from the combination with pemetrexed, mice treated with osimertinib plus cisplatin showed signs of toxicity (Fig. 2b) and treatment was stopped after 78 days. Interestingly, none of the animals treated with the drug combination showed a regrowth of tumors after treatment interruption (Fig. 2c).

The quantitative evaluation of xenograft tissue composition was morphometrically determined to assess the effect of the different therapeutic schedules on the actual tumor mass, excluding fibrosis and necrosis (Fig. 3). Thus, on Masson's trichrome stained sections, we documented that, compared to tumors from mice treated only with osimertinib, the combination of the drug with pemetrexed (Fig. 3d) or cisplatin (Fig. 3e) effectively reduced the neoplastic component of xenografts by 2.79 fold and 3.42 fold, respectively. The shrinkage in neoplastic area was coupled by a consensual increase in fibrotic tissue while necrosis did not significantly contributed to the effect of combinatory treatments.

Potential mechanisms of resistance to osimertinib were evaluated in all the tumors (eight) progressed to osimertinib. In one osimertinib-resistant tumor, $M E T$ was found amplified by FISH analysis and a higher expression level of MET was also confirmed by western blot analysis, suggesting $M E T$ amplification as a potential mechanism of resistance to osimertinib (Additional file 1: Figure S1). In the other resistant tumors (seven), analysis performed with NGS did not reveal any of the main acquired mechanisms of resistance described in the literature $[5,7]$. Tertiary acquired mutations in EGFR gene were also excluded with ddPCR, performed to avoid possible NGS false-negative results due to sensitivity limit. In all the resistant tumors the EGFR setting of parental PC9T790M clone (p.(Glu746 Ala750del) and p.T790M) was maintained. In six resistant tumors, variants in FGFR3, FOXL2, GNAQ and H3F3A, which were not present in the parental PC9T790M clone, were identified (Additional file 2: Table S1). 


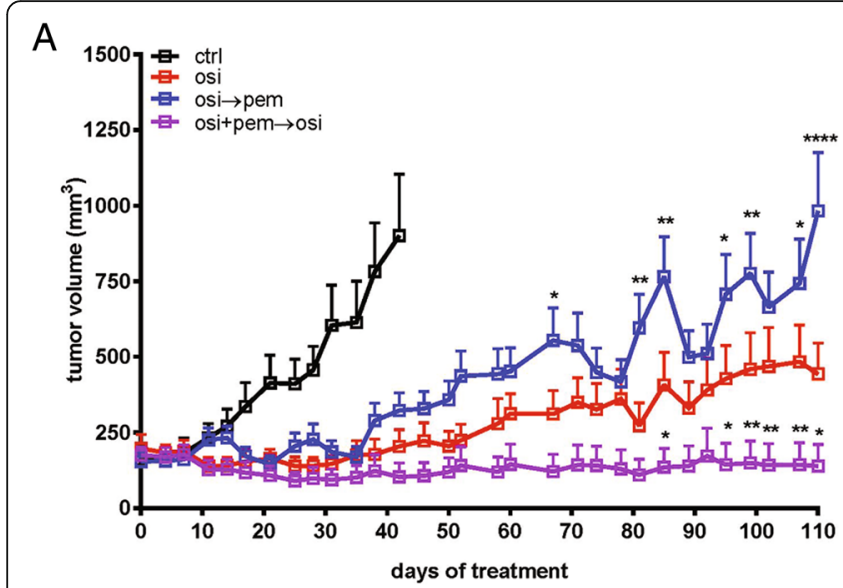

B

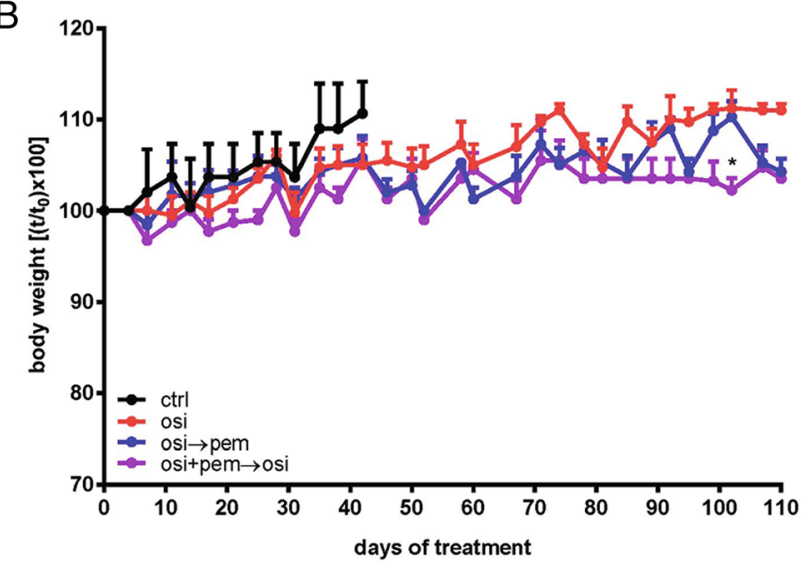

C

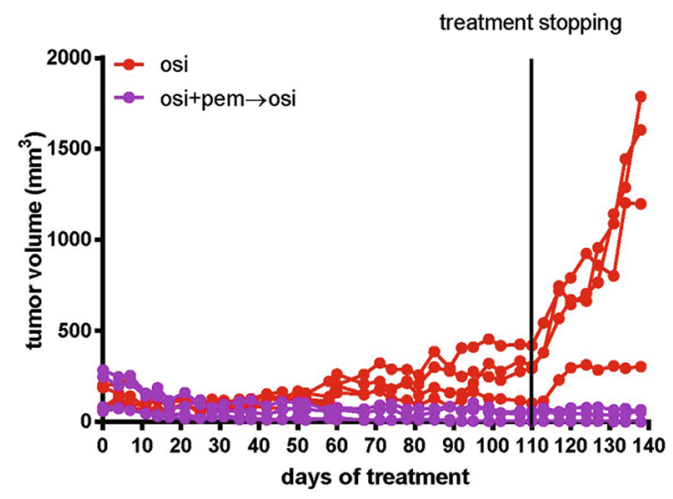

D

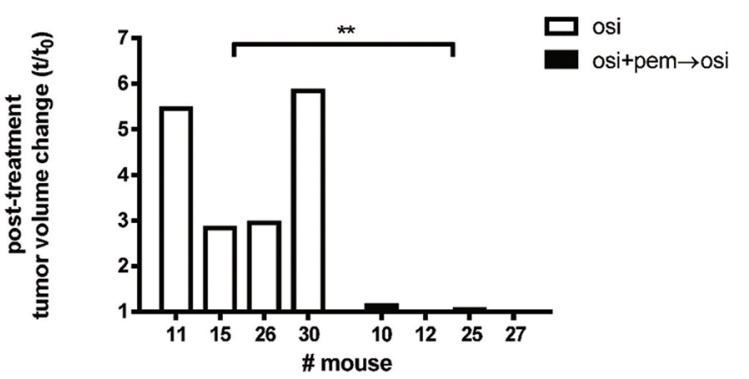

Fig. 1 Effects of osimertinib and pemetrexed association in PC9T790M xenograft model. a PC9T790M cells were subcutaneously inoculated into BALB/C nude female mice, and after tumors had reached an average size of approximately $150 \mathrm{~mm}^{3}$ the animal were treated with vehicle alone (ctrl), osimertinib (3 mg/kg once per day, five times per week) alone (osi), osimertinib intercalated every week with pemetrexed (100 mg/kg once a day, twice per week) (osi $\rightarrow$ pem), or osimertinib in combination with pemetrexed intercalated with osimertinib alone (osi + pem $\rightarrow$ osi). Tumor volume was measured twice per week and data are expressed as volume \pm SEM ( $n=8$ tumors per group). ${ }^{*} p<0.05,{ }^{* *} p<0.01,{ }^{* * *} p<0.0001$ vs osimertinib; two-way repeated measures analysis of variance followed by Bonferroni's post-test. $\mathbf{b}$ Body weight was measured twice per week and data are expressed as percent change in body weight \pm SEM ( $n=8$ tumors per group). ${ }^{*} p<0.05$ vs osimertinib; two-way repeated measures analysis of variance followed by Bonferroni's post-test. c The indicated treatments were stopped on day 110, and tumor growth was monitored for further 28 days in four animals per group. $\mathbf{d}$ Data are expressed as percent change in tumor volume: t corresponds to the tumor volume on day 138 (28 days after drug removal) and $t_{0}$ corresponds to the tumor volume on day $110 .{ }^{* *} p<0.01$; Student's $t$ test was used for the comparison of means of the two groups

The effects of osimertinib combined with pemetrexed or cisplatin on cell proliferation and cell death in PC9T790M cells in in vitro experiments were concomitantly evaluated.

As previously reported for gefitinib and pemetrexed [10], also for osimertinib the schedule of treatment in which osimertinib was given before pemetrexed or cisplatin was the less effective in inhibiting cell proliferation in long-term experiments and exerted an antagonist effect when alternated with pemetrexed (Fig. 4a). By contrast, the simultaneous treatment with osimertinib and pemetrexed or cisplatin significantly suppressed cell growth (Fig. 4a) and, as shown in Fig. 4b, enhanced cell death up to $60 \%$ in short-term experiments.

\section{Effects of pemetrexed or cisplatin on PC9T790M} osimertinib resistant clones

With the aim to test the chemosensitivity of osimertinibresistant cells, we generated two clones (osi- $\mathrm{R} \mathrm{clA}, \mathrm{clC}$ ) after 9 months of culturing PC9T790M cells in the presence of increasing concentrations of osimertinib (from 10 $\mathrm{nM}$ to $500 \mathrm{nM}$ ). Dose response treatment curves showed an $\mathrm{IC}_{50}$ for osimertinib higher than $5 \mu \mathrm{M}$ (Fig. 5a). Both clones maintained the exon 19 deletion and T790M mutation in EGFR, but neither C797S, nor amplification of $M E T$ or $H E R-2$, nor phenotypic transformation were identified as potential mechanisms of resistance (not shown). NGS analysis did not reveal any acquired mutations. Analysis of Copy Number Alteration (CNA) showed that osi- 


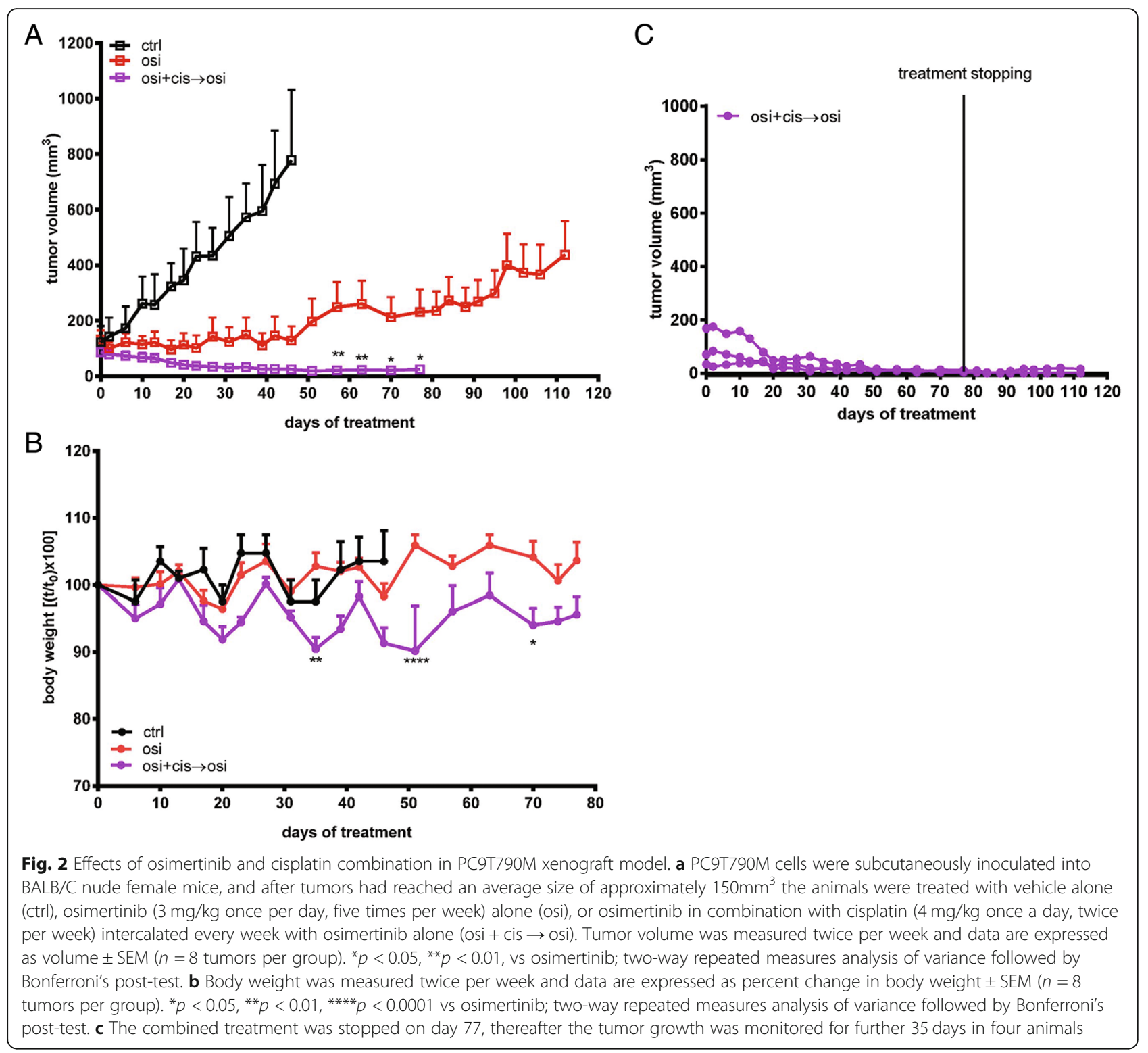

$\mathrm{R}$ clC presented an amplification of $N R A S$. This alteration was also confirmed by ddPCR CNA assay (Additional file 3: Figure S2).

Interestingly, when osimertinib was combined with pemetrexed or cisplatin no resistant clones emerged confirming results described in Fig. 4a.

As shown in Fig. 5b, c, the resistant clones were as sensitive to cisplatin as PC9T790M parental cells, but were more resistance to pemetrexed, as we previously reported also for PC9 cells resistant to gefitinib [10]. TS gene expression was significantly increased in $\mathrm{clA}$ and $\mathrm{clC}$ relative to parental cells (Fig. 5d), suggesting that this mechanism may be responsible for resistance, in keeping with published literature demonstrating that TS overexpression is a common mechanism for resistance to pemetrexed therapy in NSCLC [16]. However, we cannot rule out that other mechanisms involved in the resistance to osimertinib may also confer resistance to pemetrexed. To further confirm pemetrexed resistance in osimertinib-resistant cells, we established two cell clones from resistant tumors emerged in the osimertinib group of the experiment shown in Fig. 2a and, as expected, both cell clones showed an $\mathrm{IC}_{50}$ value for osimertinib greater than $5 \mu \mathrm{M}$ (Fig. 5e). Accordingly with the results obtained with the resistant clones generated in vitro, also the cells derived from resistant tumors were more resistant to pemetrexed (Fig. 5f).

Effects of osimertinib combined with pemetrexed or cisplatin on cell proliferation and cell death induction in PC9 and HCC827 cell lines

Considering that FDA and EMA approved osimertinib for first line treatment in EGFR mutated patients 


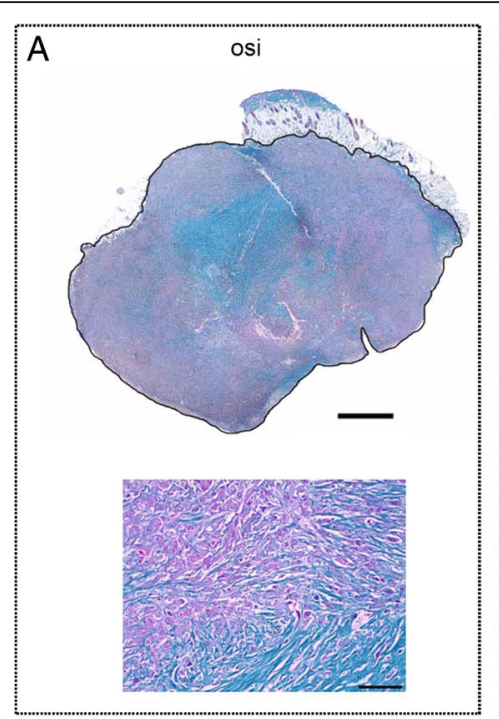

D

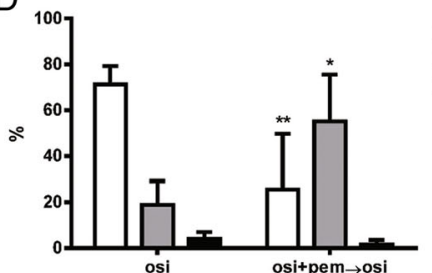

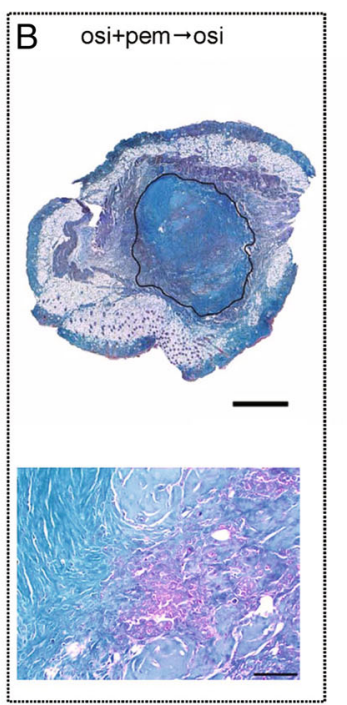

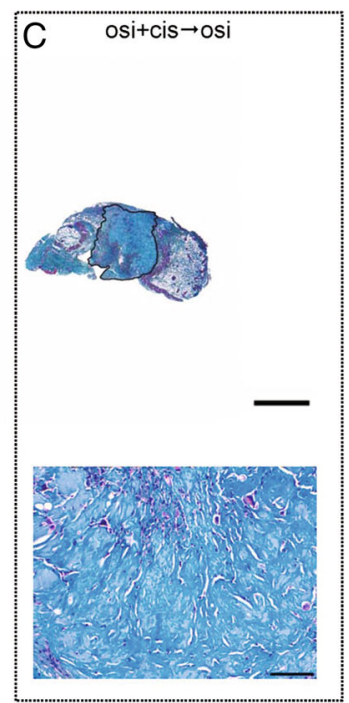

E $\square$ neoplastic tissue necrosis

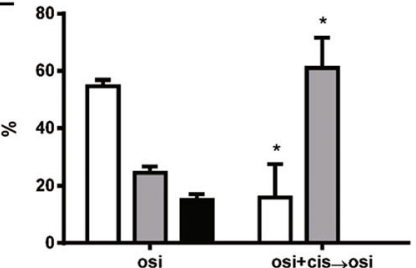

Fig. 3 Morphometric analysis of tumor xenografts. Sections from PC9T790M xenografts treated with osi (a), osi + pem $\rightarrow$ osi (b) or osi + cis $\rightarrow$ osi (c) stained by Masson's Trichrome to distinguish the fibrotic tissue (greenish) from neoplastic cells (purple). Tumors are encircled by black lines to exclude the skin, adnexa and uninvolved soft tissues. Scale bars $=1 \mathrm{~mm}$. Higher magnifications of the same samples are shown on corresponding lower panels in which the increased amount of collagen deposition after treatment with osi + pem $\rightarrow$ osi or osi + cis $\rightarrow$ osi is apparent. Scale bars $=100 \mu \mathrm{m}$. Bar graphs showing the quantitative evaluation of tissue composition (neoplastic tissue, fibrosis and necrosis) in tumor xenografts

following pharmacological treatment with osi alone or in association with pem (d) or cis (e). ${ }^{*} p<0.05,{ }^{* *} p<0.01$ vs osi group; Student's t-test

according to the results of FLAURA trial [4], we planned in vitro experiments on PC9 and HCC827 cell lines carrying EGFR mutations and sensitive to first-generation EGFR TKIs such as gefitinib and erlotinib.

Results obtained in term of inhibition of cell proliferation and induction of cell death when the cells were exposed to different treatments of osimertinib combined with pemetrexed or cisplatin (Fig. 6) were comparable to those observed for PC9T790M cells and previously described (see Fig. 4 for details). These data, even if not confirmed in xenograft models, suggest that also for naïve patients the presence of pemetrexed or cisplatin may enhance the efficacy of osimertinib in first-line treatment.

We then studied the effect of the association of osimertinib with pemetrexed or cisplatin on intracellular modulation of transduction pathways and expression of cell death regulators (Fig. 7). EGFR, AKT and ERK activation (Fig. 7a) were strongly inhibited by osimertinib both in PC9 and in PC9T790M cells as expected, being these cell lines highly responsive to osimertinib. Pemetrexed or cisplatin did not affect the phosphorylation status of these proteins and the combination with osimertinib did not differ from osimertinib alone.

By contrast, the combined treatment strongly induced the proapoptotic BCL-2 family member Bim, enhanced the activation of caspase 7 and the cleavage of PARP (Fig. 7b).

\section{Discussion}

Although preclinical and clinical researches have explored the interaction of first-generation EGFR-TKIs and cytotoxic agents $[10,17-23]$, to date there are no data on preclinical combination of chemotherapy with thirdgeneration EGFR-TKIs, such as osimertinib. The tolerability of osimertinib monotherapy indeed may allow for the development of more efficacious combination regimens.

In this study, we explored the efficacy of osimertinib combined with pemetrexed or cisplatin in NSCLC PC9T790M nude mice xenografts. A strong anti-tumor effect was observed when osimertinib was combined with pemetrexed or cisplatin intercalated, every week, with osimertinib alone. Following this approach, no tumor became resistant, differently from the treatment 


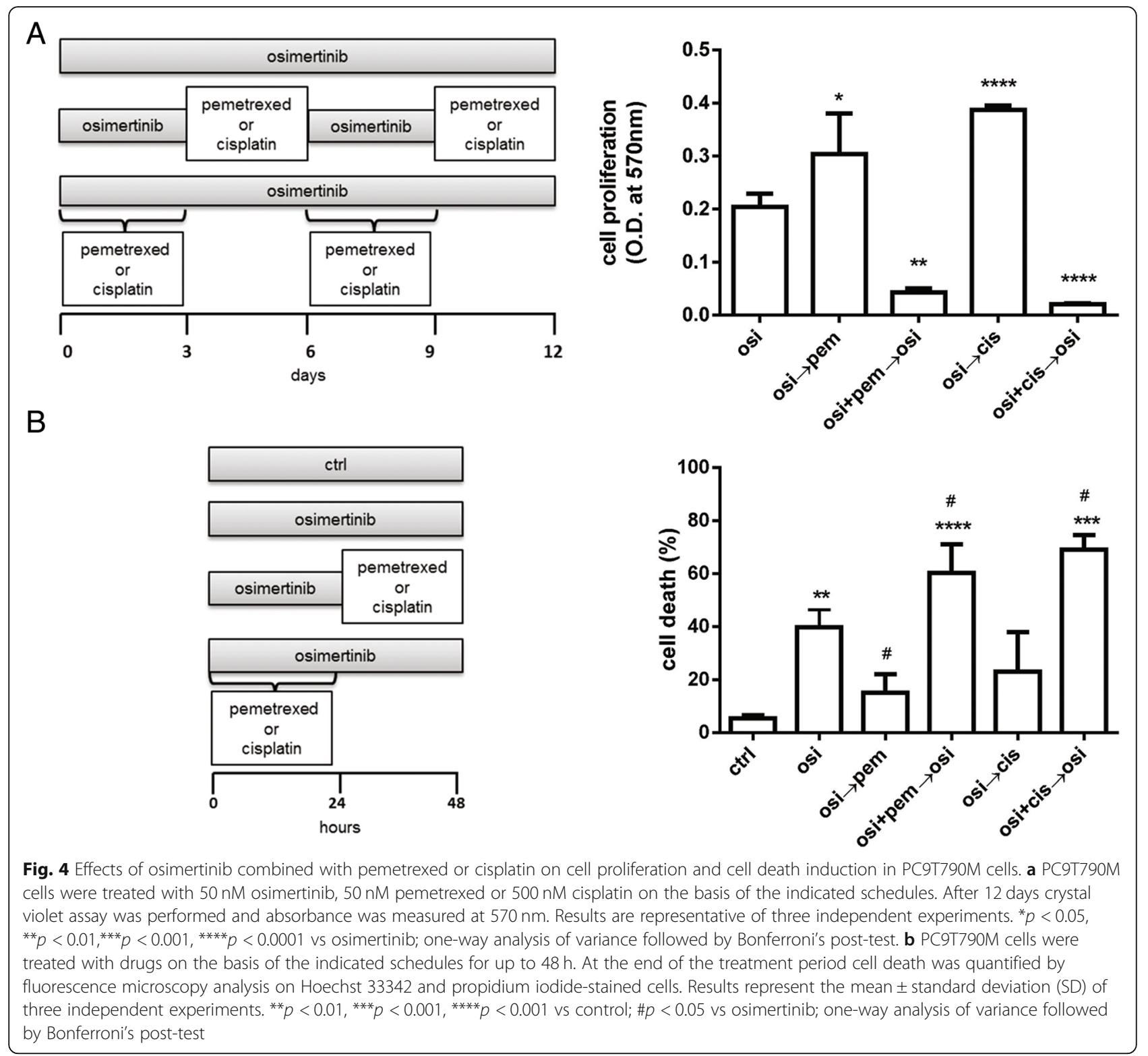

with osimertinib alone which induced acquired resistance in $50 \%$ of mice. Very interestingly, the combination treatment enhanced the percentage of fibrotic tissue within the xenograft tumors and the small tumors did not regrow when the administration of drugs was stopped, indicating a stronger efficacy in eradicating parenchymal tumor cells.

A combination of cetuximab and trastuzumab with osimertinib has been very recently reported as a new strategy for preventing resistance to osimertinib with strong and durable effects in animal studies [24, 25].

Regarding first generation EGFR TKIs, in two recent phase II randomized trials [21, 22], patients with treatment-naive advanced EGFR mutant NSCLC were treated with continuous gefitinib in combination with chemotherapy (pemetrexed in [22] and carboplatinpemetrexed in [21]). In both trials, patients on the combination with continuous gefitinib and chemotherapy experienced a statistically significant longer PFS than gefitinib alone (16.2 months versus 11.1 months in [22]; 17.5 months versus 11.9 months in [21]).

Another randomized trial evaluating a concurrent vs a sequential regimen of gefitinib and carboplatinpemetrexed in first-line [23] demonstrated a PFS and OS benefit when gefitinib was given concomitantly with chemotherapy. The sequential regimen in which gefitinib was given before chemotherapy, a schedule that in our and other preclinical studies had shown antagonistic effect $[10,19,20]$, demonstrated a worse outcome. 


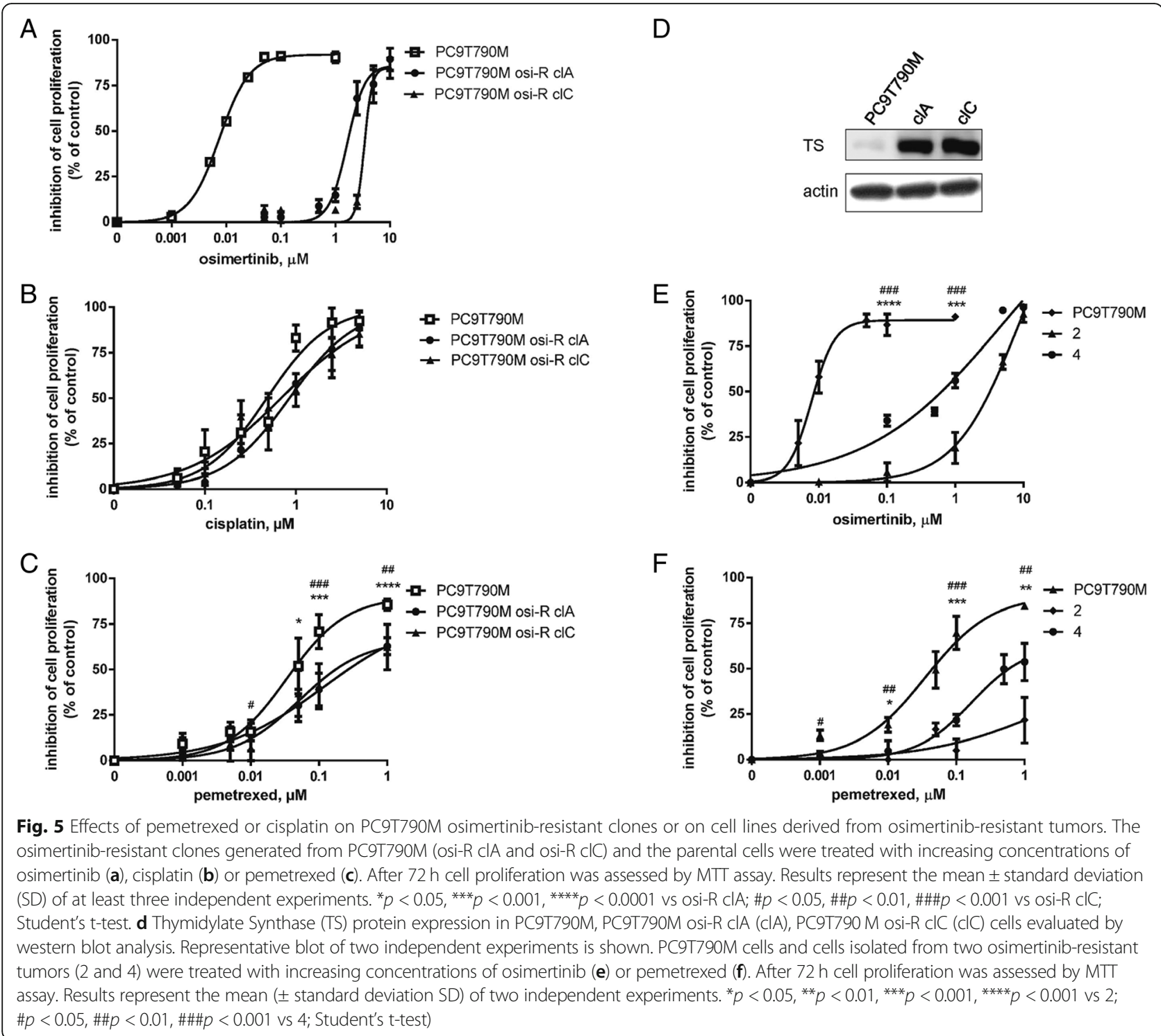

The concurrent regimen is currently being evaluated against gefitinib alone in a randomized phase III study recently presented at ESMO 2018 meeting [26]. In this trial, the patients who received a combination of gefitinib with carboplatin-pemetrexed showed a statistically significant benefit in survival (PFS of 20.9 vs 11.2 months, $p<0.001$ and OS of 52.2 vs 38.8, $p=0.013$ for gefitinib and carboplatin/pemetrexed and for gefitinib alone, respectively).

Our results indicate that, osimertinib given before pemetrexed was the worst therapy to delay resistance. By contrast, the combination of osimertinib with pemetrexed or cisplatin prevented or at least delayed the acquisition of resistance and inhibited regrowth when treatment was stopped with tumor cure. We did not observe tumor cures in the osimertinib monotherapy group, strongly indicating that the addition of chemotherapy may potentiate the efficacy of osimertinib either in term of inhibition of tumor growth or appearance of relapses.

In PC9 and PC9T790M cell lines, analysis of signaling transduction pathways and protein related to cell death revealed that the combination treatment did not affect the intracellular transduction pathways, which were already completely suppressed by osimertinib alone, but strongly enhanced apoptosis signaling via caspase-7 activation. This observation may be of relevance for the results obtained in vivo.

EGFR mutation-positive NSCLC is highly heterogeneous at the cellular level; therefore, the selective pressure exerted by TKIs may promote the clonal expansion of resistant clones through different molecular mechanisms $[27,28]$. Regardless of the resistance mechanisms that eventually will develop, it is conceivable that the enhancement in cell death associated with osimertinib/ 

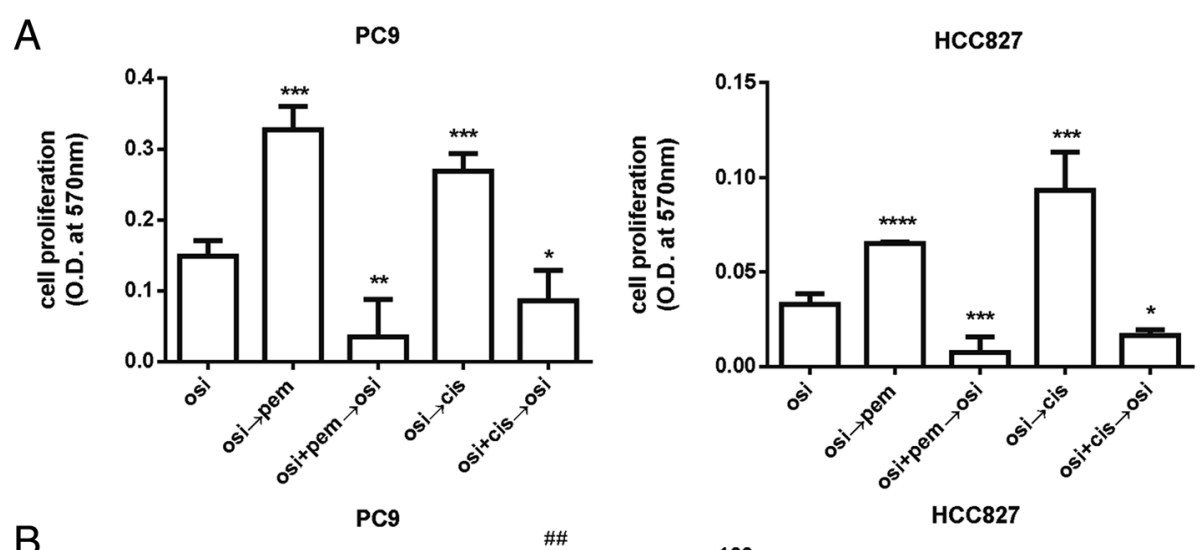

B
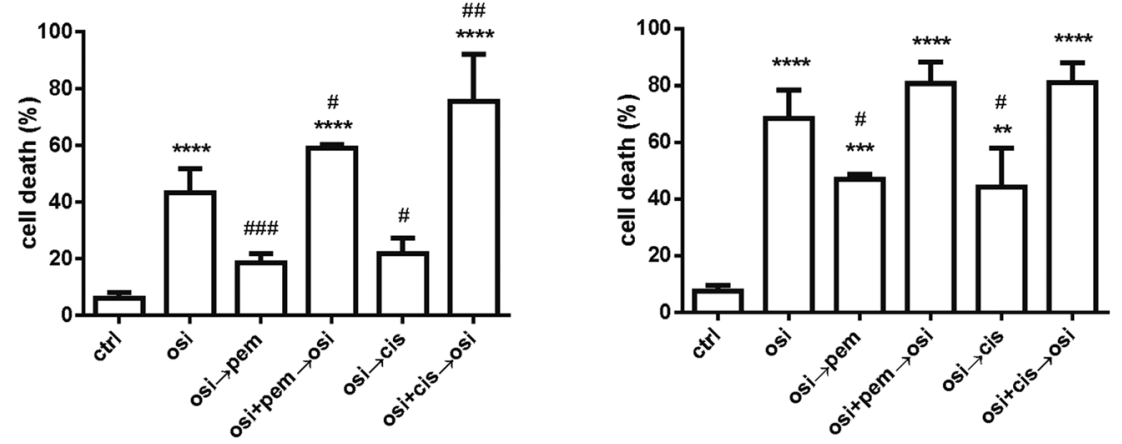

Fig. 6 Effect of osimertinib combined with pemetrexed or cisplatin on cell proliferation and cell death induction in PC9 and HCC827 cell line. a PC9 and HCC827 cells were treated with $50 \mathrm{nM}$ osimertinib, $50 \mathrm{nM}$ pemetrexed or $500 \mathrm{nM}$ cisplatin on the basis of schedules indicated in Fig. $4 a$. After 12 days crystal violet assay was performed and absorbance was measured at $570 \mathrm{~nm}$. Results are representative of three independent experiments. ${ }^{*} p<0.05,{ }^{* *} p<0.01,{ }^{* * *} p<0.001,{ }^{* * * *} p<0.0001$ vs osimertinib; one-way analysis of variance followed by Bonferroni's post-test. $\mathbf{b}$ PC9 and HCC827 cells were treated with $50 \mathrm{nM}$ osimertinib, $50 \mathrm{nM}$ pemetrexed or $500 \mathrm{nM}$ cisplatin on the basis of the schedules indicated in Fig. 4b. At the end of the treatment period cell death was quantified by fluorescence microscopy analysis on Hoechst 33342 and propidium iodide-stained cells. Results represent the mean ( \pm standard deviation SD) of three independent experiments. ${ }^{* * *} p<0.001$ vs control; \#p $<0.05$, \#\#p $<0.01$, \#\#\# $<0.001$ vs osimertinib; one-way analysis of variance followed by Bonferroni's post-test

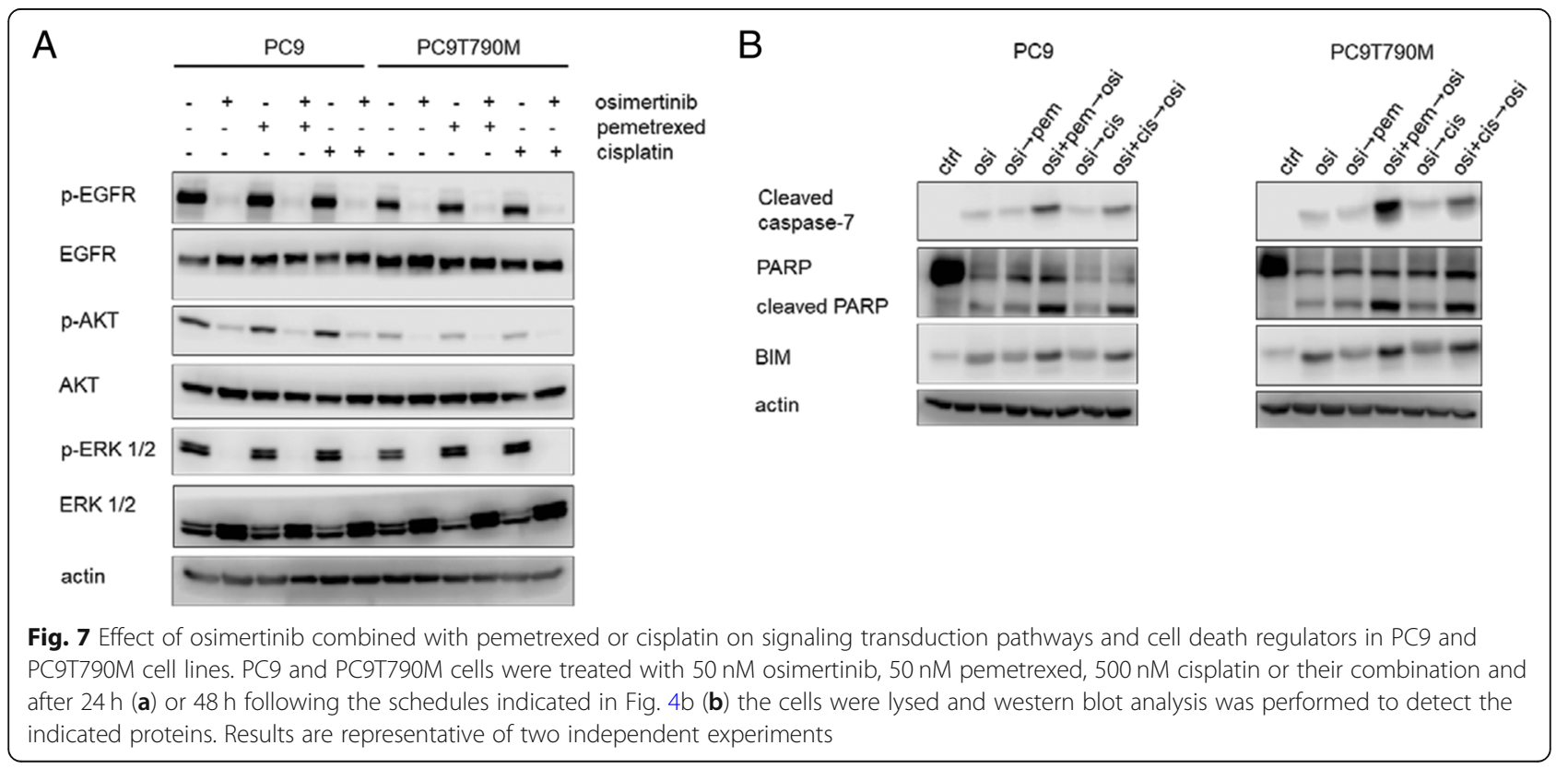


chemotherapy combination, presumably triggered also in vivo, may postpone the emergence of resistance to EGFR-TKIs by limiting the original tumor heterogeneity. Then, a delay in the clonal expansion of pre-existing resistant clones may reasonably explain the results from in vivo experiments. However, it is worth noting that chemotherapy itself exerts a selective pressure that may influence tumor heterogeneity and subsequent clonal evolution. Therefore, despite we cannot conclude that the combined approach could completely prevent the resistance, in the timing we performed the experiments no resistant tumors developed when mice were treated with osimertinib combined with pemetrexed or cisplatin.

For patients with T790M positive tumors progressing after second-line osimertinib, chemotherapy remains the standard of care. However, our data indicate that osimertinib resistant clones, obtained after 9 months of selection with increasing concentrations of the drug or isolated from osimertinib resistant xenografts, were less sensitive to pemetrexed with respect to parental cells. These clones showed a marked increase in TS expression, which reduces the efficacy of pemetrexed [16].

Differently, the responsiveness to cisplatin remained unchanged. These results suggest to use a chemotherapy regimen not-containing pemetrexed in patients progressing after osimertinib; no clinical data are available about the best chemotherapy regimen in this setting.

Potential mechanisms of resistance were evaluated in all the tumors progressed to treatment and in resistant cell lines. Apart from $M E T$ amplification, detected in one tumor, we have not been able to identify other already known mechanisms responsible for the lack of responsiveness to osimertinib. Nevertheless, in resistant tumors we reported (see Additional files) SNV and/or CNA detected by NGS analysis, but actually little is known about their involvement in mechanisms of acquired resistance to osimertinib and this aspect remains a matter for further investigation. FGFR3, observed in one tumor, was described only as a partner in oncogenic fusion, no missense variants have been reported until now as mediators of resistance [29]. NRAS amplification, observed in osi- $\mathrm{R} \mathrm{clC}$, could potentially be a mechanism of resistance to osimertinib. Indeed, this CNA was observed as mechanism of resistance in vitro to naquotinib, a third-generation EGFR inhibitor [30]. FOXL2, GNAQ and $H 3 F 3 A$ have not been reported in the literature as associated with acquired resistance to osimertinib, and these variants will undergo functional characterization to elucidate their potential role.

In view of the superior efficacy of osimertinib in the first-line treatment [4], we tested in vitro, in PC9 and HCC827 cell lines, the combination between osimertinib and pemetrexed or cisplatin. The advantage in using the combinatory regimen was demonstrated also in these cell lines harbouring EGFR activating mutation without T790M, suggesting that cytotoxic agents should be added to EGFR-TKIs as first-line therapy in advanced NSCLC with activating EGFR mutation.

\section{Conclusion}

Our preclinical results provide a strong rationale for randomized studies comparing osimertinib vs osimertinib plus chemotherapy, either in EGFR T790M positive and also in EGFR-TKI naïve NSCLC patients. In particular, a phase III trial evaluating osimertinib combined with platinum-pemetrexed vs. osimertinib alone could be the right step forward to significantly prolong the survival of EGFR-mutated NSCLC patients.

\section{Additional files}

Additional file 1: Figure S1. MET analysis in a tumor progressed to osimertinib. (TIF $873 \mathrm{~kb}$ )

Additional file 2: Table S1. Analysis of putative mechanism of acquired resistance to osimertinib: Next Generation Sequencing results. (TIFF 13 kb)

Additional file 3: Figure S2. ddPCR assay of NRAS amplification in osimertinib-resistant clones generated from PC9T790M. (TIFF 1494 kb)

\section{Abbreviations}

CNA: Copy Number Alteration; EGFR: Epidermal Growth Factor Receptor; NSCLC: Non-Small Cell Lung Cancer; PFS: Progression-Free Survival;

SNV: Single Nucleotide Variation; TKIs: Tyrosine Kinase Inhibitors; TS: Thymidylate Synthase

\section{Acknowledgements}

Not applicable

\section{Authors' contributions}

SLM, DC, MB and AC conducted the cell biology and molecular biology experiments. SLM, LF and EB performed in vivo experiments. GD and MG isolated neoplastic cells from xenograft tumors. RM and AR performed NGS and digital droplet PCR analyses. DM, AF and FQ performed morphometric analysis of tumor xenografts. CAL and AS performed FISH analysis. RA and PGP analyzed results. CF performed statistical analysis. RA and MT wrote the paper. SLM and PGP edited the manuscript. All authors contributed to revise the manuscript and approved the final version for publication.

\section{Funding}

This work was supported by AIRC (Italian Association for Cancer Research), Milan (grant IG2017-20074 PI M. Tiseo); AstraZeneca, Milan Italy; Associazione Augusto per la Vita (Novellara, RE), Fondazione Martalive ONLUS (Brugherio, MI). The funders had no role in study design, data collection and analysis, decision to publish, or preparation of the manuscript.

\section{Availability of data and materials}

Data and materials will be shared.

Ethics approval and consent to participate

All experiments involving animals and their care were performed with the approval of the Local Ethical Committee of University of Parma (Organismo per la Protezione e il Benessere degli Animali) and by the Italian Ministry of Health, in accordance with the institutional guidelines that are in compliance with national (D.Lgs. 26/2014) and international (Directive 2010/63/EU) laws and policies.

Consent for publication

Not applicable. 


\section{Competing interests}

No potential conflicts of interest were disclosed.

Advisory boards and speakers' fee for Astra-Zeneca (M. Tiseo).

\section{Author details}

'Department of Medicine and Surgery, University of Parma, Parma, Italy. ${ }^{2}$ Medical Oncology Unit, University Hospital of Parma, Parma, Italy. ${ }^{3}$ Food and Drug Department, University of Parma, Parma, Italy. ${ }^{4}$ Italian Workers' Compensation Authority (INAIL) Research Center, Parma, Italy. ${ }^{5}$ Center of Excellence for Toxicological Research (CERT), University of Parma, Parma, Italy. ${ }^{6}$ Centre for Integrative Biology (CIBIO), University of Trento, Trento, Italy.

Received: 9 January 2019 Accepted: 21 May 2019

Published online: 28 May 2019

\section{References}

1. Pao W, Miller VA, Politi KA, Riely GJ, Somwar R, Zakowski MF, Kris MG, Varmus $\mathrm{H}$. Acquired resistance of lung adenocarcinomas to gefitinib or erlotinib is associated with a second mutation in the EGFR kinase domain. PLoS Med. 2005;2:e73.

2. Mok TS, Wu YL, Ahn MJ, Garassino MC, Kim HR, Ramalingam SS, Shepherd FA, He Y, Akamatsu $H$, Theelen WS, et al. Osimertinib or platinumPemetrexed in EGFR T790M-positive lung Cancer. N Engl J Med. 2017;376: 629-40.

3. Janne PA, Yang JC, Kim DW, Planchard D, Ohe Y, Ramalingam SS, Ahn MJ, Kim SW, Su WC, Horn L, et al. AZD9291 in EGFR inhibitor-resistant nonsmall-cell lung cancer. N Engl J Med. 2015;372:1689-99.

4. Soria JC, Ohe Y, Vansteenkiste J, Reungwetwattana T, Chewaskulyong B, Lee KH, Dechaphunkul A, Imamura F, Nogami N, Kurata T, et al. Osimertinib in untreated EGFR-mutated advanced non-small-cell lung Cancer. N Engl J Med. 2018;378:113-25.

5. Minari R, Bordi P, Del Re M, Facchinetti F, Mazzoni F, Barbieri F, Camerini A, Comin $C E$, Gnetti L, Azzoni C, et al. Primary resistance to osimertinib due to SCLC transformation: issue of T790M determination on liquid re-biopsy. Lung Cancer. 2018;115:21-7.

6. Ramalingam SS, Yang JC, Lee CK, Kurata T, Kim DW, John T, Nogami N, Ohe $Y$, Mann $H$, Rukazenkov $Y$, et al. Osimertinib as first-line treatment of EGFR mutation-positive advanced non-small-cell lung Cancer. J Clin Oncol. 2018; 36:841-9.

7. Yang Z, Yang N, Ou Q, Xiang Y, Jiang T, Wu X, Bao H, Tong X, Wang X, Shao $Y W$, et al. Investigating novel resistance mechanisms to third-generation EGFR tyrosine kinase inhibitor Osimertinib in non-small cell lung Cancer patients. Clin Cancer Res. 2018;24:3097-107.

8. Papadimitrakopoulou VA, Wu Y-L, Han J-Y, Ahn M-J, Ramalingam SS, John T, Okamoto I, Yang JC-H, Bulusu KC, Laus G, et al. Analysis of resistance mechanisms to osimertinib in patients with EGFR T790M advanced NSCLC from the AURA3 study. Ann Oncol. 2018;29:mdy424.064.

9. Ramalingam SS, Cheng Y, Zhou C, Ohe Y, Imamura F, Cho BC, Lin M-C, Majem M, Shah R, Rukazenkov $Y$, et al. Mechanisms of acquired resistance to first-line osimertinib: preliminary data from the phase III FLAURA study. Ann Oncol. 2018;29:mdy424.063.

10. La Monica S, Madeddu D, Tiseo M, Vivo V, Galetti M, Cretella D, Bonelli M, Fumarola C, Cavazzoni A, Falco A, et al. Combination of Gefitinib and Pemetrexed prevents the acquisition of TKI resistance in NSCLC cell lines carrying EGFR-activating mutation. J Thorac Oncol. 2016;11:1051-63.

11. Planchard D, Popat S, Kerr K, Novello S, Smit EF, Faivre-Finn C, Mok TS, Reck M, Van Schil PE, Hellmann MD, Peters S. Metastatic non-small cell lung cancer: ESMO clinical practice guidelines for diagnosis, treatment and follow-up. Ann Oncol. 2018:29:iv192-237.

12. La Monica S, Caffarra C, Saccani F, Galvani E, Galetti M, Fumarola C, Bonelli M, Cavazzoni A, Cretella D, Sirangelo R, et al. Gefitinib inhibits invasive phenotype and epithelial-mesenchymal transition in drug-resistant NSCLC cells with MET amplification. PLoS One. 2013;8:e78656.

13. Cavazzoni A, Alfieri RR, Carmi C, Zuliani V, Galetti M, Fumarola C, Frazzi R, Bonelli M, Bordi F, Lodola A, et al. Dual mechanisms of action of the 5benzylidene-hydantoin UPR1024 on lung cancer cell lines. Mol Cancer Ther. 2008;7:361-70

14. Chmielecki J, Foo J, Oxnard GR, Hutchinson K, Ohashi K, Somwar R, Wang L, Amato KR, Arcila M, Sos ML, et al. Optimization of dosing for EGFR-mutant non-small cell lung cancer with evolutionary cancer modeling. Sci Transl Med. 2011;3:90ra59.
15. La Monica S, Cretella D, Bonelli M, Fumarola C, Cavazzoni A, Digiacomo G, Flammini L, Barocelli E, Minari R, Naldi N, et al. Trastuzumab emtansine delays and overcomes resistance to the third-generation EGFR-TKI osimertinib in NSCLC EGFR mutated cell lines. J Exp Clin Cancer Res. 2017; 36:174.

16. Takezawa K, Okamoto I, Okamoto W, Takeda M, Sakai K, Tsukioka S, Kuwata K, Yamaguchi H, Nishio K, Nakagawa K. Thymidylate synthase as a determinant of pemetrexed sensitivity in non-small cell lung cancer. $\mathrm{Br}$ J Cancer. 2011;104:1594-601.

17. Cui J, Zhang Y, Su D, Li T, Li Y. Efficacy of combined icotinib and pemetrexed in EGFR mutant lung adenocarcinoma cell line xenografts. Thorac Cancer. 2018;9:1156-65

18. Feng $X$, Zhang Y, Li T, Li Y. Sequentially administrated of pemetrexed with icotinib/erlotinib in lung adenocarcinoma cell lines in vitro. Oncotarget. 2017:8:114292-9.

19. Giovannetti E, Lemos C, Tekle C, Smid K, Nannizzi S, Rodriguez JA, Ricciard S, Danesi R, Giaccone G, Peters GJ. Molecular mechanisms underlying the synergistic interaction of erlotinib, an epidermal growth factor receptor tyrosine kinase inhibitor, with the multitargeted antifolate pemetrexed in non-small-cell lung cancer cells. Mol Pharmacol. 2008;73:1290-300.

20. Li T, Ling YH, Goldman ID, Perez-Soler R. Schedule-dependent cytotoxic synergism of pemetrexed and erlotinib in human non-small cell lung cancer cells. Clin Cancer Res. 2007;13:3413-22.

21. Han B, Jin B, Chu T, Niu Y, Dong Y, Xu J, Gu A, Zhong H, Wang H, Zhang X, et al. Combination of chemotherapy and gefitinib as first-line treatment for patients with advanced lung adenocarcinoma and sensitive EGFR mutations: a randomized controlled trial. Int J Cancer. 2017;141:1249-56.

22. Yang JC, Cheng Y, Murakami H, Yang P, He J, Nakagawa K, Kang JH, Kim J, Wnag X, Enatsu S, et al: Gefitinib with or without Pemetrexed in nonsquamous (NS) non-small cell lung Cancer (NSCLC) with EGFR mutation (Mut): final overall survival results from a randomized phase II trial. Ann Oncol 2018, 29:viii493-viii547. 4https://doi.org/10.1093/annonc/mdy1292.

23. Oizumi S, Sugawara S, Minato K, Harada T, Inoue A, Fujita Y, Maemondo M, Watanabe S, Ito K, Gemma A, et al. Updated survival outcomes of NEJ005/ TCOG0902, a randomized phase II study of concurrent (C) versus sequential alternating (S) gefitinib and chemotherapy in previously untreated nonsmall cell lung cancer (NSCLC) with sensitive epidermal growth factor receptor (EGFR) mutations. J Clin Oncol. 2017;35.

24. Romaniello D, Mazzeo L, Mancini M, Marrocco I, Noronha A, Kreitman M, Srivastava S, Ghosh S, Lindzen M, Salame TM, et al. A combination of approved antibodies overcomes resistance of lung Cancer to Osimertinib by blocking bypass pathways. Clin Cancer Res. 2018.

25. Mancini M, Gal H, Gaborit N, Mazzeo L, Romaniello D, Salame TM, Lindzen M, Mahlknecht G, Enuka Y, Burton DG, et al. An oligoclonal antibody durably overcomes resistance of lung cancer to third-generation EGFR inhibitors. EMBO Mol Med. 2018;10:294-308.

26. Seike M, Inoue A, Sugawara S, Morita S, Hosomi Y, Ikeda S, Watanabe K, Takahashi K, Fujita Y, Harada T, et al. Phase III study of gefitinib (G) versus gefitinib-carboplatin-pemetrexed (GCP) as first-line treatment for patients (pts) with advanced non-small cell lung cancer (NSCLC) with EGFR mutations (NEJ009). Ann Oncol. 2018;29:mdy292.005.

27. Yu HA, Suzawa K, Jordan E, Zehir A, Ni A, Kim R, Kris MG, Hellmann MD, Li BT, Somwar R, et al. Concurrent alterations in EGFR-mutant lung cancers associated with resistance to EGFR kinase inhibitors and characterization of mTOR as a mediator of resistance. Clin Cancer Res. 2018;24:3108-18.

28. Blakely CM, Watkins TBK, Wu W, Gini B, Chabon JJ, McCoach CE, McGranahan N, Wilson GA, Birkbak NJ, Olivas VR, et al. Evolution and clinical impact of co-occurring genetic alterations in advanced-stage EGFR-mutant lung cancers. Nat Genet. 2017:49:1693-704.

29. Ou SI, Horn L, Cruz M, Vafai D, Lovly CM, Spradlin A, Williamson MJ, Dagogo-Jack I, Johnson A, Miller VA, et al. Emergence of FGFR3-TACC3 fusions as a potential by-pass resistance mechanism to EGFR tyrosine kinase inhibitors in EGFR mutated NSCLC patients. Lung Cancer. 2017;111:61-4.

30. Ninomiya K, Ohashi K, Makimoto G, Tomida S, Higo H, Kayatani H, Ninomiya T, Kubo T, Ichihara E, Hotta K, et al. MET or NRAS amplification is an acquired resistance mechanism to the third-generation EGFR inhibitor naquotinib. Sci Rep. 2018;8:1955.

\section{Publisher's Note}

Springer Nature remains neutral with regard to jurisdictional claims in published maps and institutional affiliations. 